\title{
A child with severe congenital cytomegalovirus infection
}

\author{
A C D de Alwis ${ }^{1}$, C Liyanage ${ }^{2}$, A H M M Jayaweera ${ }^{2}$, S P Abeywardene ${ }^{1}$
}

Sri Lanka Journal of Child Health, 2008; 37: 95-97

(Key words: child, congenital cytomegalovirus infection)

Cytomegalovirus (CMV) is the most common cause of congenital infection in the world, affecting approximately $0.3 \%$ to $2.4 \%$ of all pregnancies ${ }^{1}$. When congenital rubella is virtually eliminated by the ongoing immunization programme, congenital CMV has the potential to emerge as an important public health problem in Sri Lanka. Congenital CMV is clinically important as it can lead to major long term sequelae like cerebral palsy and blindness, and in fact, it is the commonest infectious cause of sensorineural deafness ${ }^{2}$. We describe a child with severe congenital CMV infection.

\section{Case History}

A 33 year old primigravid mother from Ratnapura delivered a baby boy weighing $1650 \mathrm{~g}\left(<0.4^{\text {th }}\right.$ centile $)$ at term by an elective caesarean section, following a pregnancy complicated by intrauterine growth retardation. The length and head circumference were also below $0.4^{\text {th }}$ centile. He was noted to have multiple ecchymoses on the trunk and lower extremities at birth. No other bleeding diatheses were evident and he was not dysmorphic. Liver and spleen were palpable 2 and $4 \mathrm{~cm}$ below the costal margin, but there was no lymphadenopathy. Rest of the examination was unremarkable. Mother had received the rubella vaccine prior to conception and no antenatal history of a febrile illness or blood transfusion was noted.

$\overline{{ }^{1} \text { Consultant Paediatrician, }{ }^{2} \text { Registrar in Paediatrics, }}$ General Hospital, Ratnapura, Sri Lanka

(Received on 26 June 2007. Accepted on 20 July 2007)
Blood film showed a hypochromic microcytic anaemia and a marked thrombocytopenia. Platelet counts were persistently below $10 \times 10^{9} / \mathrm{L}$. Liver functions were deranged with elevated AST and ALT, and kidney functions were normal. Semilateral skull radiograph showed intracranial calcifications that conform to the shape of the ventricles (Figure 1), and head ultrasound scan revealed multiple cortical calcifications and fullness of the ventricular system. Periventricular, subependymal and basal ganglia calcification and dilated lateral and third ventricles were seen in the cranial CT (Figure 2). Chest radiograph was normal. ELISA test for CMV IgM of the mother and baby and PCR test for CMV genome of the baby were positive. Rubella and toxoplasma antibodies of both mother and infant were negative. Bilateral macular scars of the retina and pallor of left optic disc were noted on detailed ophthalmological examination. The newborn was managed symptomatically with repeated platelet transfusions, intravenous antibiotics until the cultures were confirmed to be negative, and routine care of a small for gestational age newborn.

On follow up, his growth was retarded, and development was severely delayed in all skill areas. He was developing spastic quadriplegic type of cerebral palsy. He was referred for intensive physiotherapy and speech and language therapy. At 18 months, he developed generalized tonic clonic seizures which were treated with sodium valproate. Brain Stem Evoke Resposes (BSER) showed bilateral sensorineural deafness. He was also visually impaired with loss of central vision and preservation of some peripheral vision. 


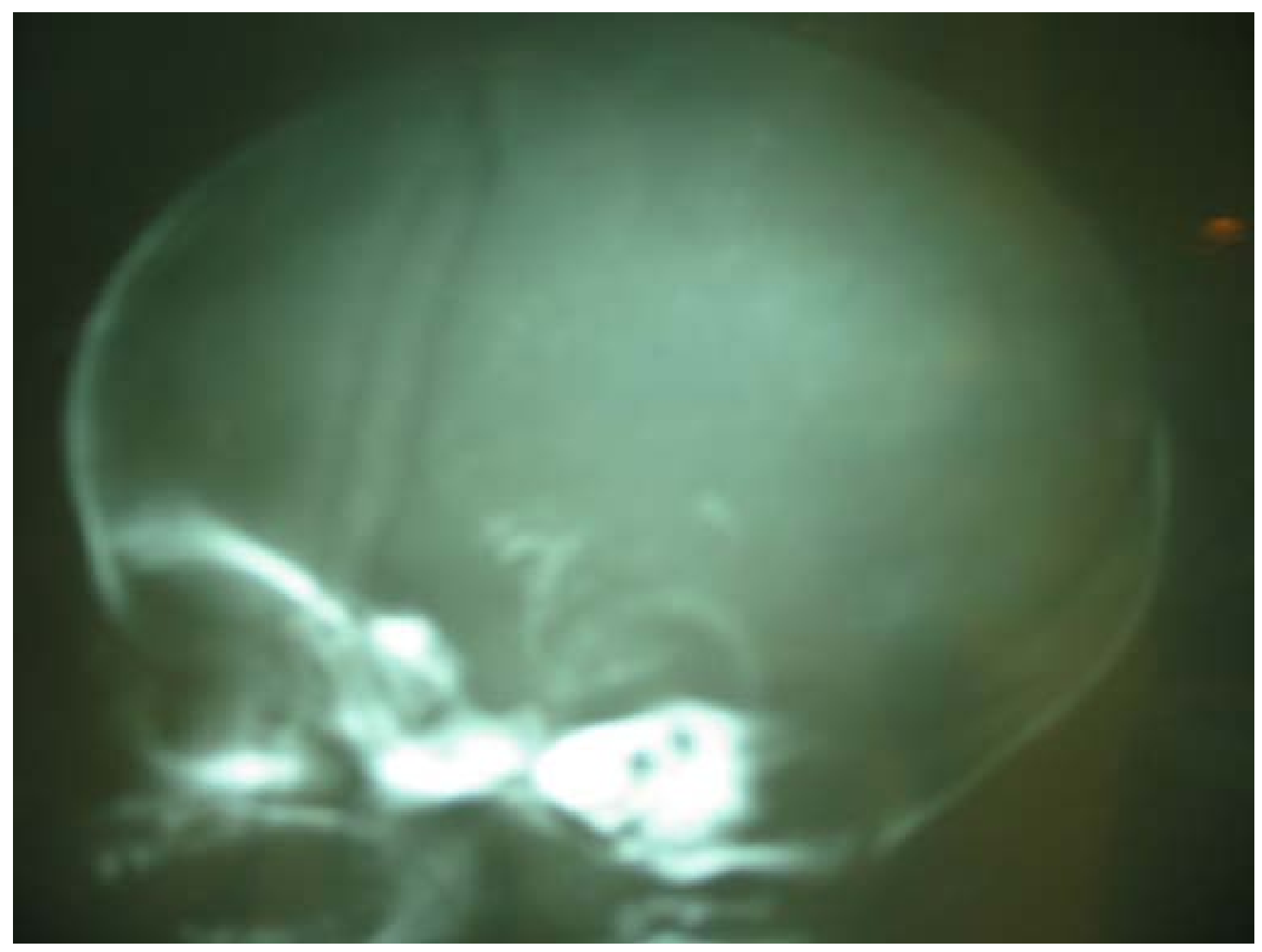

Figure 1 Lateral skull radiograph showing intracranial calcifications that conform to the shape of the ventricles.

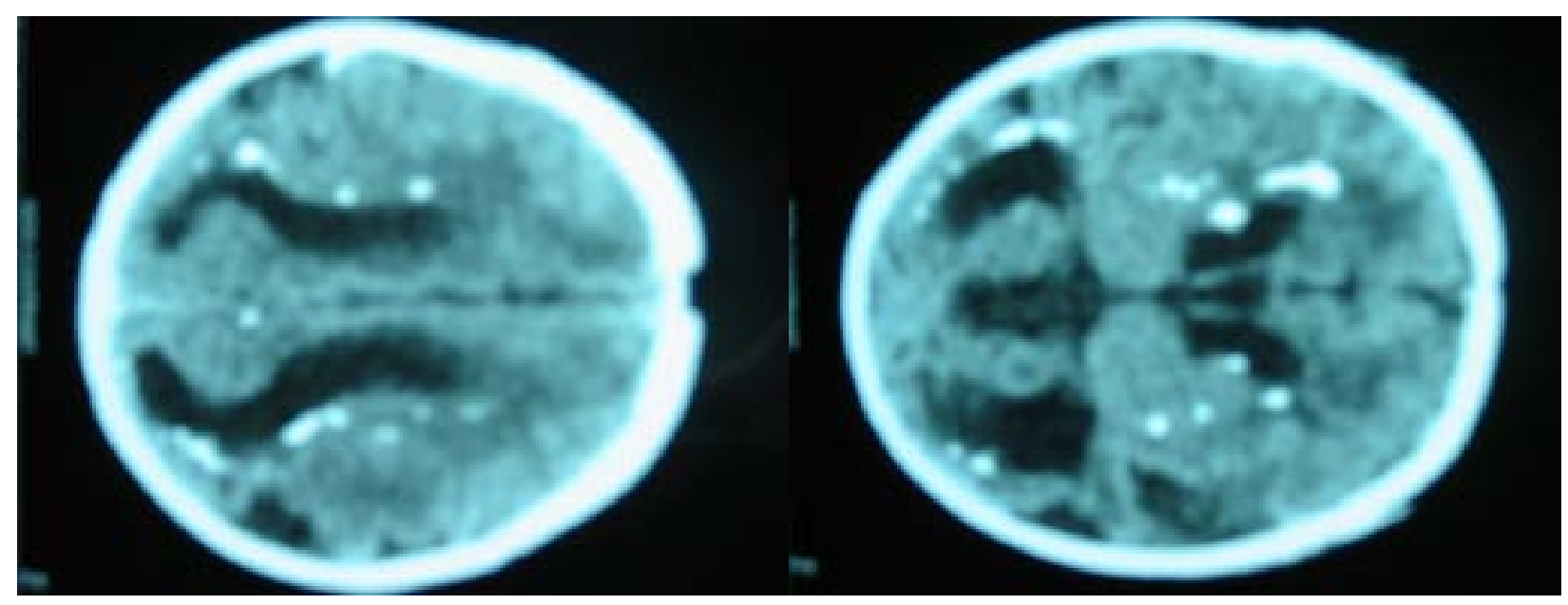

Figure 2 CT scan of the brain showing parenchymal calcifications particularly in subependymal and periventricular regions and dilatation of ventricles 


\section{Discussion}

CMV is a DNA virus and a member of the herpesviridae family. 'CMV inclusion disease' or congenital CMV infection is caused by vertical transmission of human cytomegalovirus from mother to infant. This could occur transplacentally, or during the perinatal period via cervico-vaginal secretions or breast milk. While ninety percent of the infected remain asymptomatic, ten percent develop symptoms involving multiple organs ${ }^{3}$. Infants born to mothers who are infected early in pregnancy may be more likely to be small for gestational age and to have neurological manifestations like microcephaly and intracranial calcification. Those who acquire CMV after birth may have features of acute visceral disease like pneumonia, hepatosplenomegaly, hepatitis, thrombocytopenia, and atypical lymphocytosis ${ }^{3}$. Our patient probably acquired the infection during early pregnancy.

Although congenital CMV is known to follow both primary and recurrent maternal infections, it is common to occur following a primary infection of the mother ${ }^{4}$. Positive IgM antibodies for CMV in our mother makes primary maternal infection more likely.

Management of symptomatic congenital CMV infection is mainly supportive. The place for platelet transfusion in newborns with non-immunologically mediated thrombocytopenia is not clearly defined. As a general rule platelets should be given to thrombocytopenic neonates when the degree of thrombocytopenia alone or in combination with other complications results in an unacceptable risk of haemorrhage ${ }^{5}$. A randomized, controlled trial has shown that ganciclovir therapy begun in the neonatal period in infants with congenital CMV disease involving the central nervous system prevents hearing deterioration at 6 months and possibly at 1 year of age ${ }^{6}$. It is now offered to all neonates with $\mathrm{CNS}$ involvement due to proven congenital CMV infection in some developed countries. Unfortunately, ganciclovir is not currently available in Sri Lanka.

Congenital CMV is preventable. Pregnant mothers acquire the infection by exposing themselves to the saliva or urine of young children. Good personal hygiene, especially hand-washing, with soap and warm water can reduce the risk of CMV acquisition ${ }^{7}$. In fact, American College of Obstetricians and Gynecologists recommends that physicians should counsel pregnant women about preventing CMV acquisition through careful attention to personal hygiene. A CMV vaccine is in the pipeline. Until it becomes freely available, effective educational interventions to inform pregnant mothers about congenital CMV prevention may be the only way forward for us in the developing world.

\section{References}

1. Trincado D E, Rawlison W D. Congenital and perinatal infection with cytomegalovirus. $J$ Paediatr Child Health 2001; 37: 187-92.

2. Nance W, Lim B, Dodson K. Importance of congenital cytomegalovirus infections as a cause for pre-lingual hearing loss. Journal of Clinical Virology 2006; 35 (2), 221-5.

3. Munro S C, Trincado D, Hall B. Symptomatic infant characteristics of congenital cytomegalovirus disease in Australia. J Paediatr Child Health 2005; 41 (8): 449-52.

4. Stagno S, Pass R F et al. Primary cytomegalovirus infection in pregnancy. Incidence, transmission to fetus, and clinical outcome. JAMA. 1986; 256:1904-8.

5. Roberts I, Murray N A. Neonatal thrombocytopenia: causes and management. Archives of Disease in Childhood Fetal and Neonatal Edition 2003; 88: F359-64.

6. Kimberlin D W, Lin C Y et al. Effect of ganciclovir therapy on hearing in symptomatic congenital cytomegalovirus disease involving the central nervous system: a randomized, controlled trial. J Pediatr. 2003; 143(1):16-25.

7. Cannon M J, Davis K F, Washing our hands of the congenital cytomegalovirus disease epidemic. BMC Public Health. 2005; 5: 70. 